\title{
Ultrastructural Basis for Interactions Between Central Opioids and Catecholamines. II. Nuclei of the Solitary Tracts
}

\author{
Virginia M. Pickel, June Chan, and Teresa A. Milner \\ Department of Neurology and Division of Neurobiology, Cornell University Medical College, New York, New York 10021
}

Interactions between central opioids and catecholamines are thought to underlie the ability of adrenergic agonists both to lower blood pressure and alleviate certain symptoms of opiate withdrawal. We examined the cellular substrate for interactions between neurons containing enkephalin-like opioid peptides and catecholamines in cardiovascular portions of the medial nuclei of the solitary tracts (m-NTS) of adult rats. Single sections were dually labeled using a double-bridged peroxidase method for the localization of a monoclonal leucine (Leu ${ }^{5}$ )-enkephalin-antibody and immunoautoradiography for the localization of polyclonal antibodies against the catecholamine-synthesizing enzyme tyrosine hydroxylase (TH). Light microscopy revealed a few perikarya and numerous varicosities containing Leus-enkephalin-like immunoreactivity (LE-LI). These were distributed among TH-labeled perikarya and processes throughout the rostrocaudal NTS. Electron microscopy of the m-NTS at the level of the area postrema further established the single as well as dual localization of TH and LE- $\mathrm{TI}$ in individual perikarya, dendrites, and axon terminals. Silver grains indicative of TH-labeling were usually distributed throughout the cytoplasm, whereas the peroxidase reaction product for LE-LI was localized principally to large (80-150 $\mathrm{nm}$ ), densecore vesicles. Immunoautoradiographic labeling for TH was detected in 118 terminals within a series of sections containing 183 terminals with LE-LI. Of these, $26 \%$ of the THlabeled terminals and $32 \%$ of the enkephalin-containing terminals formed symmetric synapses with unlabeled dendrites, while only $7 \%$ of each type formed symmetric synapses with TH-labeled dendrites. In favorable planes of sections, the unlabeled as well as TH-labeled dendrites received convergent input from both types of terminals. A few of the remaining terminals that contained either TH or LE-LI formed asymmetric junctions with unlabeled distal dendrites; the others were without recognizable synaptic specializations within the plane of section. Approximately $20 \%$ of the THlabeled terminals and $6 \%$ of the terminals containing LE-LI were dually labeled for both antibodies. These were invested with astrocytic processes characterized by bundles of intermediate filaments. We conclude that within cardiovas-

\footnotetext{
Received Sept. 14, 1988; revised Jan. 9, 1989; accepted Jan. 10, 1989.

We gratefully acknowledge the support provided by grants from NIH (HL18974), NIMH (MH00078 and MH40342), and NIDA (DA04600). We are also grateful for the TH-antiserum provided by Dr. C. Abate (Roche Institute of Molecular Biology) and for the photographic help of Joan Chun.

Correspondence should be addressed to Dr. Virginia M. Pickel, Division of Neurobiology, Cornell University Medical College, 411 East 69th Street, New York, NY 10021.

Copyright (C) 1989 Society for Neuroscience $0270-6474 / 89 / 072519-17 \$ 02.00 / 0$
}

cular portions of the m-NTS, opioid peptides and catecholamines contained within the same or separate terminals modulate the activity of target neurons through direct symmetric, probably inhibitory, synaptic junctions and may additionally modulate the activity of neighboring astrocytes through exocytotic release from large dense-core vesicles. The detection of TH immunoreactivity in a few neurons postsynaptic to terminals containing LE-LI and/or TH indicates that one type of receptive neuron is catecholaminergic. However, the vast majority of the neurons receptive to opioid and/or catecholaminergic terminals in the $\mathrm{m}$-NTS contain an unidentified transmitter.

Opiates and adrenergic agonists exert hypotensive actions that are at least partially mediated through direct synaptic modulation of adrenergic neurons in the $\mathrm{Cl}$ area (Hökfelt et al., 1974) of the rostral ventrolateral medulla (Granata et al., 1983; Milner et al., 1989). The comparably dense distribution of binding sites for opiates and alpha $\mathrm{a}_{2}$-adrenoreceptors in portions of the medial nuclei of the solitary tract (m-NTS) known to receive cardiovagal afferents additionally suggests that some of the cardiovascular effects of opiates and catecholamines may involve neurons in the solitary nuclei (Atweh and Kuhar, 1977; Kalia and Sullivan, 1982; Kuhar, 1982; Donoghue et al., 1984; Dashwood ct al., 1985). Microinjections of alpha $\mathrm{a}_{2}$-adrenergic agonists such as clonidine in the intermediate and caudal m-NTS elicit hypotensive effects (Lipski and Solnicka, 1976), while opiates may elicit either decreases or increases in blood pressure depending upon the concentration and receptor specificity of the applied opiates (Hassen et al., 1982, 1983). Additionally, iontophoretic application of opiate peptides in discrete hypothalamic sites and in the m-NTS elicits sympathoexcitation (Pfeiffer et al., 1983; Appel et al., 1986). At least some of the differences in physiological responses to opiate agonists in the m-NTS may be attributed to the transmitters and efferent pathways of the receptive neurons. Noradrenergic neurons of the A2-group (Dahlström and Fuxe, 1965) are thought to be one of the targets of terminals containing endogenous opioid peptides in the caudal m-NTS (Pickel et al., 1979). This conclusion is based on morphological similarities between terminals immunocytochemically labeled for methionine $\left(\mathrm{Mct}^{5}\right)$-cnkephalin and tcrminals forming synapses with dendrites immunolabeled for the catecholamine-synthesizing enzyme tyrosine hydroxylase $(\mathrm{TH})$ in adjacent sections (Pickel et al., 1979). However, the synaptic junctions onto catecholaminergic neurons from enkephalin-labeled terminals were never confirmed in single sections. Moreover, other types of associations such as coexistence or convergent in put to common target neurons could not be examined with the methods used in the earlier study (Pickel et al., 1979). We have addressed 


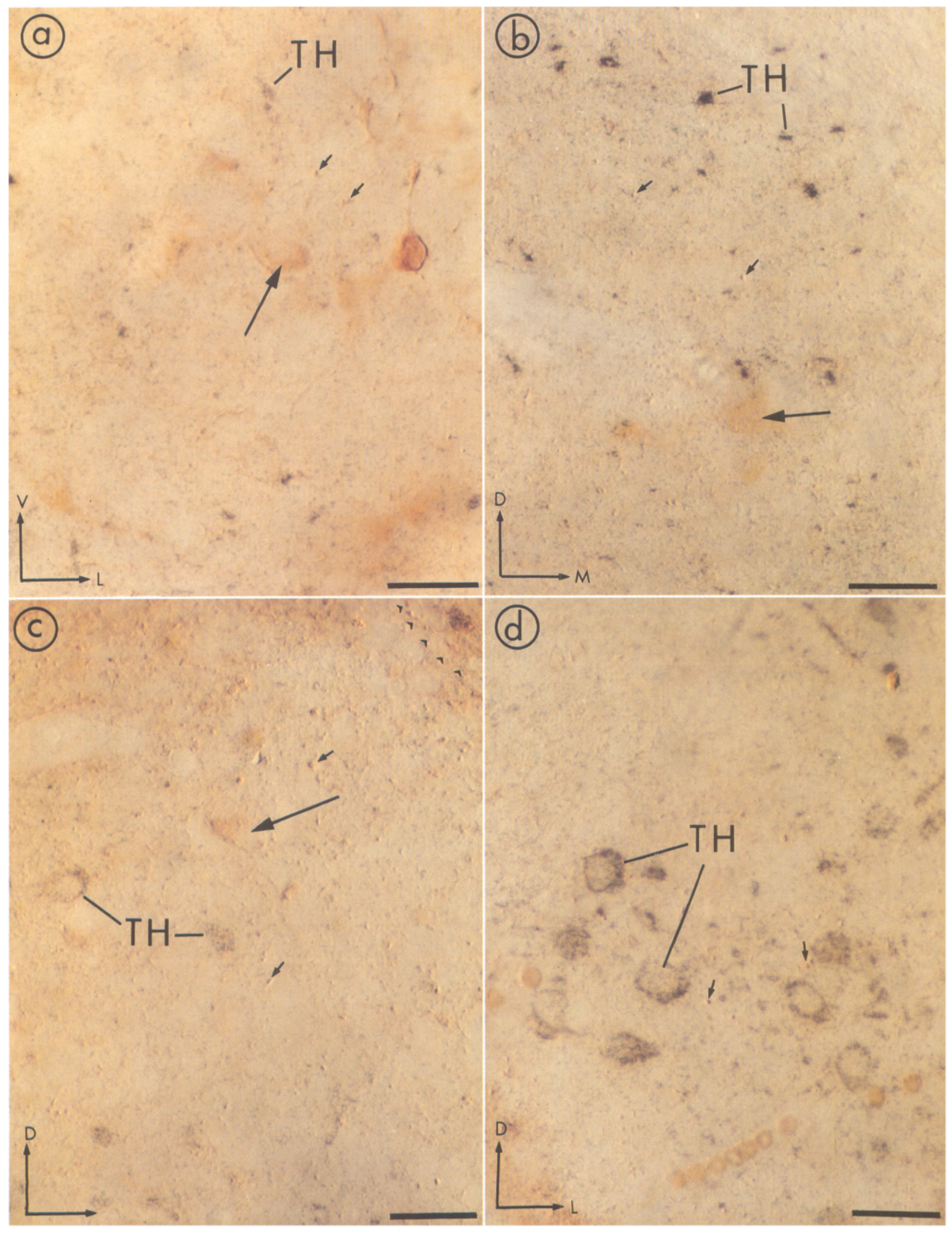


these questions by combining immunoautoradiographic and immunoperoxidase methods to localize rabbit $\mathrm{TH}$ and rat leucine $\left(\mathrm{Leu}^{5}\right.$ )-enkephalin (LE) antibodies in single sections through the $\mathrm{m}$-NTS of colchicine-treated adult rats. A quantitative ultrastructural evaluation of the relative frequency of detection of different types of associations revealed that unlabeled, sometimes common, target neurons received the major input from terminals singly labeled for either LE or TH and from those dually labeled for both of these markers.

\section{Materials and Methods}

Details of the methods used in this study have been described (Pickel et al., 1986a; Milner et al., 1989). Thus, only a brief description is given. Fifteen adult (180-250 gm) male Sprague-Dawley rats were anesthetized with halothane $\left(2 \%\right.$ in $\left.100 \% \mathrm{O}_{2}\right)$ and given intraventricular injections of colchicine ( $100 \mu \mathrm{g} / 7.5 \mu \mathrm{l}$ saline). Twenty-four hours later they were perfused through the ascending aorta sequentially with (1) $10 \mathrm{cc}$ heparinsaline, (2) $50 \mathrm{cc}$ of $3.75 \%$ acrolein and $2 \%$ paraformaldehyde in $0.1 \mathrm{M}$ phosphate buffer ( $\mathrm{pH} 7.4$ ), and (3) $200 \mathrm{cc}$ of $2 \%$ paraformaldehyde in $0.1 \mathrm{M}$ phosphate buffer. Coronal $30-40 \mu \mathrm{m}$ Vibratome sections were collected through the rostrocaudal extent of the solitary tract nuclei (Paxinos and Watson, 1986). The sections were placed in $1 \%$ sodium borohydride in $0.1 \mathrm{M}$ phosphate buffer for $30 \mathrm{~min}$ and then rinsed in phosphate buffer prior to immunocytochemical labeling.

A mouse monoclonal antibody raised against $\mathrm{Leu}^{5}$-enkephalin was commercially obtained from Sera Lab (Crawley Down, United Kingdom) and was tested for cross-reactivity by immunoblots and by adsorption with $\mathrm{Leu}^{5}$ - and $\mathrm{Met}^{5}$-enkephalin, $\beta$-endorphin, and various dynorphin $A$ fragments. The cross-reactions were principally against Leu $^{5}$ - and Met ${ }^{5}$-enkephalin [see Milner et al. (1989) for methods and cross-reactivity results]. Rabbit polyclonal antibodies against trypsintreated TH were produced and characterized as previously described (Joh and Ross, 1983).

Vibratome sections were first incubated for 18-24 hr in a mixture of the TH- and LE-antibodies at dilutions of 1:2000 and 1:200, respectively. These were washed in $0.1 \mathrm{M}$ Tris-saline and then incubated for $1 \mathrm{hr}$ in a 1:100 dilution of ${ }^{125}$ I-labeled donkey anti-rabbit immunoglobulin (Amersham, Arlington Heights, IL) at a 1:100 dilution of a solution having a radioactive concentration of $100 \mu \mathrm{Ci} / \mathrm{ml}$ and a specific activity of $17 \mu \mathrm{Ci} / \mu \mathrm{g}$. These sections then were washed extensively over several hours in Tris-saline and processed by a double-bridged peroxidase-antiperoxidase (PAP) labeling method using anti-mouse reagents for the localization of the mouse, LE antibody (Ordronncau ct al., 1981; Milner et al., 1989). These sections then were processed for light microscopic autoradiography or were embedded in Epon 812 between 2 sheets of Aclar plastic (Masurovsky and Bunge, 1968), cut on an ultramicrotome and processed for electron microscopic autoradiography (Beaudet, 1982; Pickel et al., 1986a).

Controls. To evaluate the possible labeling of the first antibody by antisera in the second stage of the dual-labeling procedure, some sections were processed for dual labeling with omission of either the first or second primary antibody. In this case, detection of only the one appropriate label was considered as evidence that there was little, if any, nonspecific cross-reactions. [See Milner et al. (1989) for adsorption controls for LE-antibody and Joh and Ross (1983) and Pickel (1981), for TH-antiserum.] Additionally, cross-reactivity between LE and TH antibodies was shown to be negligible by adsorption controls. The addition of $50 \mu \mathrm{g} \mathrm{LE}$ to $0.5 \mathrm{ml}$ of 1:2000 dilution of TH antibody did not diminish detected immunoreactivity.

Sampling. Light microscopic sections dually labeled for LE and TH were examined throughout the rostrocaudal NTS. Electron microscopic analysis was carricd out on 7 plastic-cmbedded and dually labeled sections collected through the m-NTS at the level of the area postrema from 4 animals (see Fig. $2 b$ for area sampled). These sections were chosen based on the optimal preservation of morphological details and maximal detection of TH- and LE-like immunoreactivities as observed by both light and electron microscopy. In electron micrographs, specific autoradiographic labeling was differentiated from nonspecific (background) labeling following identification of silver grains over the suspected source of radiation in at least 2 and sometimes 3 or more adjacent thin sections. Additionally, a quantitative analysis using a modification of the method of Salpeter et al. (1977) was performed in the rostral ventrolateral medulla of sections from the same brains as the NTS samples were taken (see Milner et al., 1989).

\section{Results}

\section{Light microscopy}

Light microscopy revealed a differential labeling between the brown PAP-reaction product for LE-LI and black silver grains indicative of immunoautoradiographic labeling for TH-I (Fig. 1). Both labels were detected in a few perikarya and processes in sections examined throughout the rostrocaudal extent of the NTS. However, the relative abundance of immunoreactive perikarya, their distribution, and intensity of labeling for either $\mathrm{TH}$ or for LE-LI varied markedly at different rostrocaudal levels or even between adjacent cells within the same section (Fig. 1). The more intensely labeled and more abundant perikarya containing LE-LI were located in the medial and lateral portions of rostral NTS (Figs. $1, a, b ; 2 a$ ). At this level, most of the THlabeled perikarya, largely representing the C2-group of adrenergic neurons (Hökfelt et al., 1974), were located near the medial borders of the NTS. These were dorsal and medial to most of the perikarya containing LE-LI. However, large transected processes, presumably proximal dendrites, as well as smallcr varicosities showed accumulations of silver grains indicative of $\mathrm{TH}$ laheling (Figs. $1, a, b ; 2 a$ ). In the m-NTS at the level of area postrema, peroxidase-labeled processes containing LE-LI were far more numerous than the similarly labeled perikarya (Fig. $1 c$ ). Perikarya within the more dorsal m-NTS (Figs. $1 c, 2 b$ ) were more lightly labeled for TH. A few other cells in this area showed peroxidase reaction for LE-LI without overlying silver grains. Some of the TH-labeled perikarya with low densities of silver grains also appeared to contain low levels of peroxidase product; however, the differential labeling within single cells could not be resolved by light microscopy. The TH-immunoreactive (I) perikarya within the more ventral subdivision of the caudal

Figure 1. Photomicrographs showing the dual localization of tyrosine hydroxylase $(T H)$ and LE-LI in the m-NTS. $a$, In ventral and lateral portions of the m-NTS corresponding to the level shown in Figure $2 a$, low-intensity brown peroxidase reaction product for LE-LI is seen in a perikaryon (large arrow) just adjacent to a more intensely peroxidase labeled cell. Peroxidase labeling is also seen in a number of varicose processes (small arrows). Accumulations of silver grains indicate TH-I in transected processes. $b$. In the more dorsal and medial portions of the m-NTS at the same level as in $a$, numerous TH-I processes are dispersed among other varicose processes (small arrows) and a perikaryon (large arrow) containing low-intensity peroxidase reaction for LE-LI. $c$, In the dorsomedial portions of the $\mathrm{m}$-NTS at the same level as show in the schematic in Figure $2 b$, relatively low levels of immunoautoradiographic labeling for TH-I and peroxidase reaction for LE-LI (large arrow) are seen in a small group of perikarya located ventral and lateral to the area postrema. A portion of the ventral boundary of the area postrema is indicated by the dashed line in the upper right of the figure. Peroxidase positive varicosities (small arrows) appear more abundant near the area postrema. $d$, In the more ventral $\mathrm{m}-\mathrm{NTS}$ at the same level as in $c$, intense immunoautoradiographic labeling for TH is seen in numerous perikarya and processes. Varicose processes (small arrows) but not perikarya show peroxidase labeling for LE-LI. All sections used for $a-c$ were immunocytochemically labeled within the same containers and the autoradiographs were examined after a $5 \mathrm{~d}$ autoradiographic exposure. Interference contrast optics. Arrows indicate the orientation of the photomicrograph within the section. $D$, dorsal; $V$, ventral; $M$, medial; and $L$, lateral. Scale bar, $50 \mu \mathrm{m}$. 
(a)

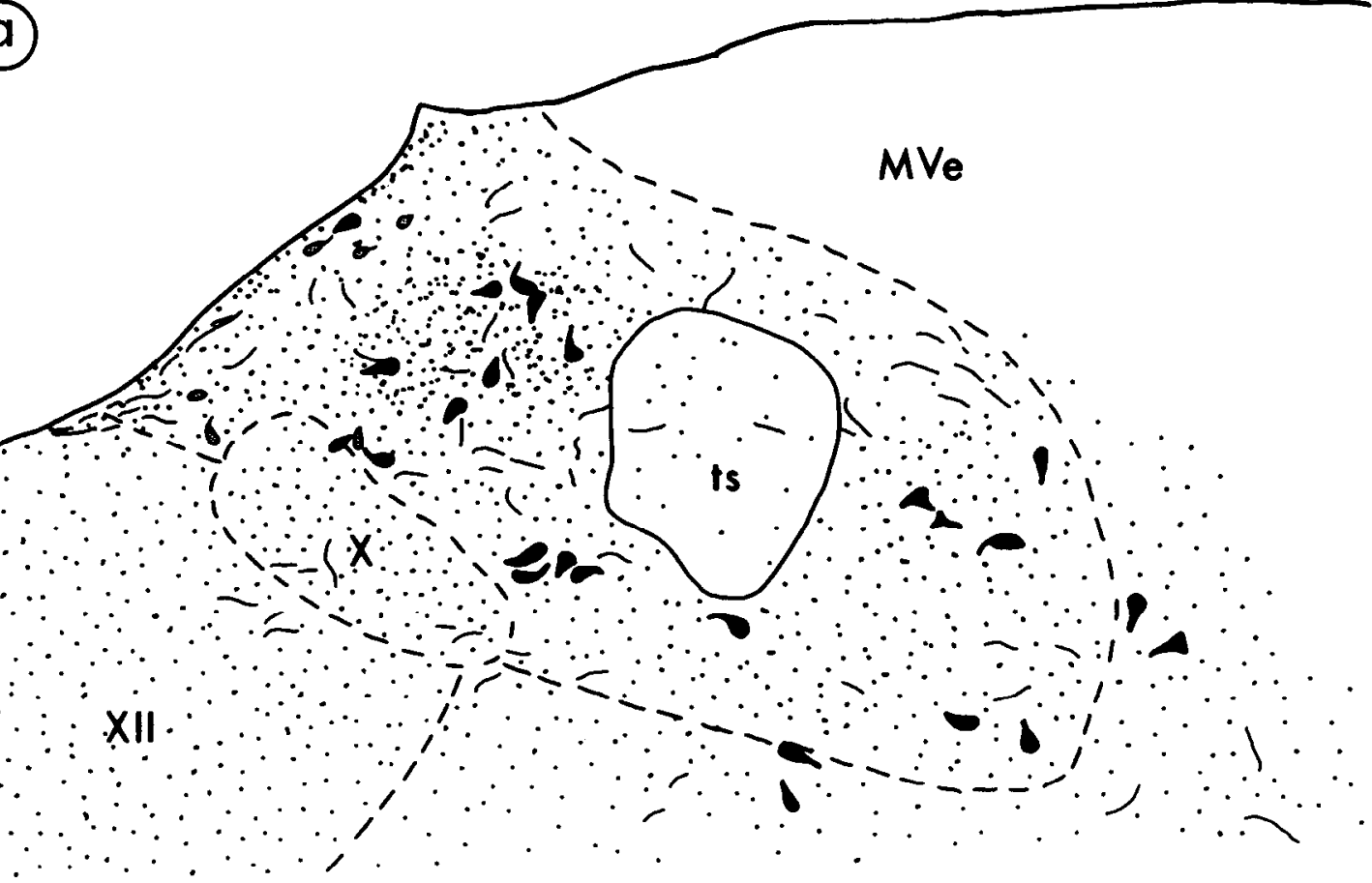

(b)

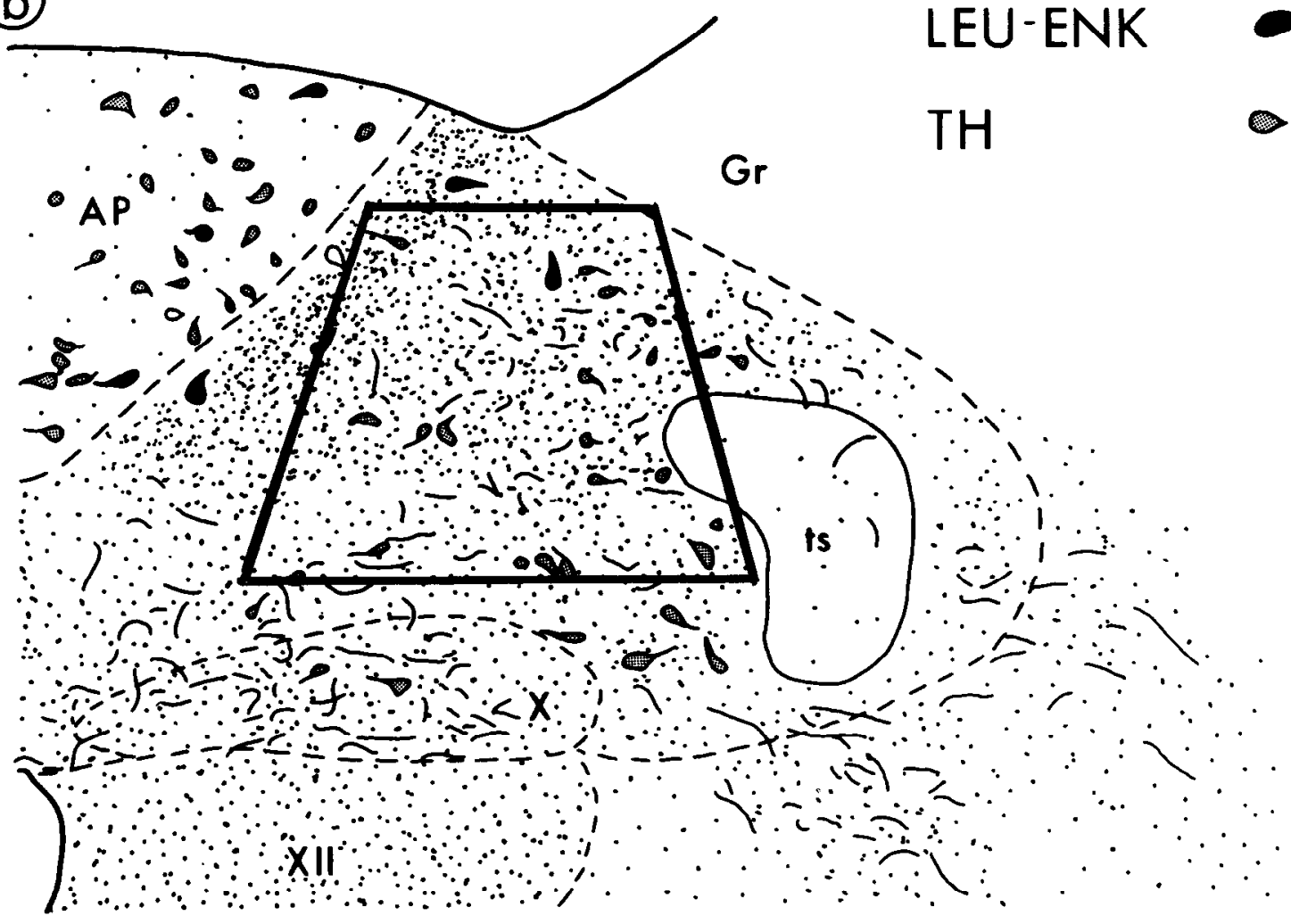

Figure 2. Schematic drawing showing the distribution of perikarya and processes immunoreactive for either LE-LI or TH. a, Rostral NTS level shows a cluster of peroxidase (LE-LI)-labeled perikarya located medially with respect to the tractus solitarius $(t s)$. Other peroxidase-labeled perikarya also are seen ventrolaterally to the tract. In contrast, the TH-labeled perikarya are located more dorsally near the ventricular lumen. $b$, Intermediate NTS at the level of the area postrema $(A P)$ shows peroxidase-labeled (LE-LI) perikarya located in the more dorsal m-NTS toward the base of the AP. A few TH-labeled perikarya also are seen within the same region. However, the majority of the TH-labeled cells are seen more ventrally, above and also sometimes within the motor nucleus of the vagus $(X)$. Pyramid shows area sampled for electron microscopy. $X$, dorsal motor nucleus of the 10th cranial nerve and $X I I$, the twelfth; $M V e$, medial vestibular; $G r$, gracilis nuclei. Scale bar, $100 \mu \mathrm{m}$. 


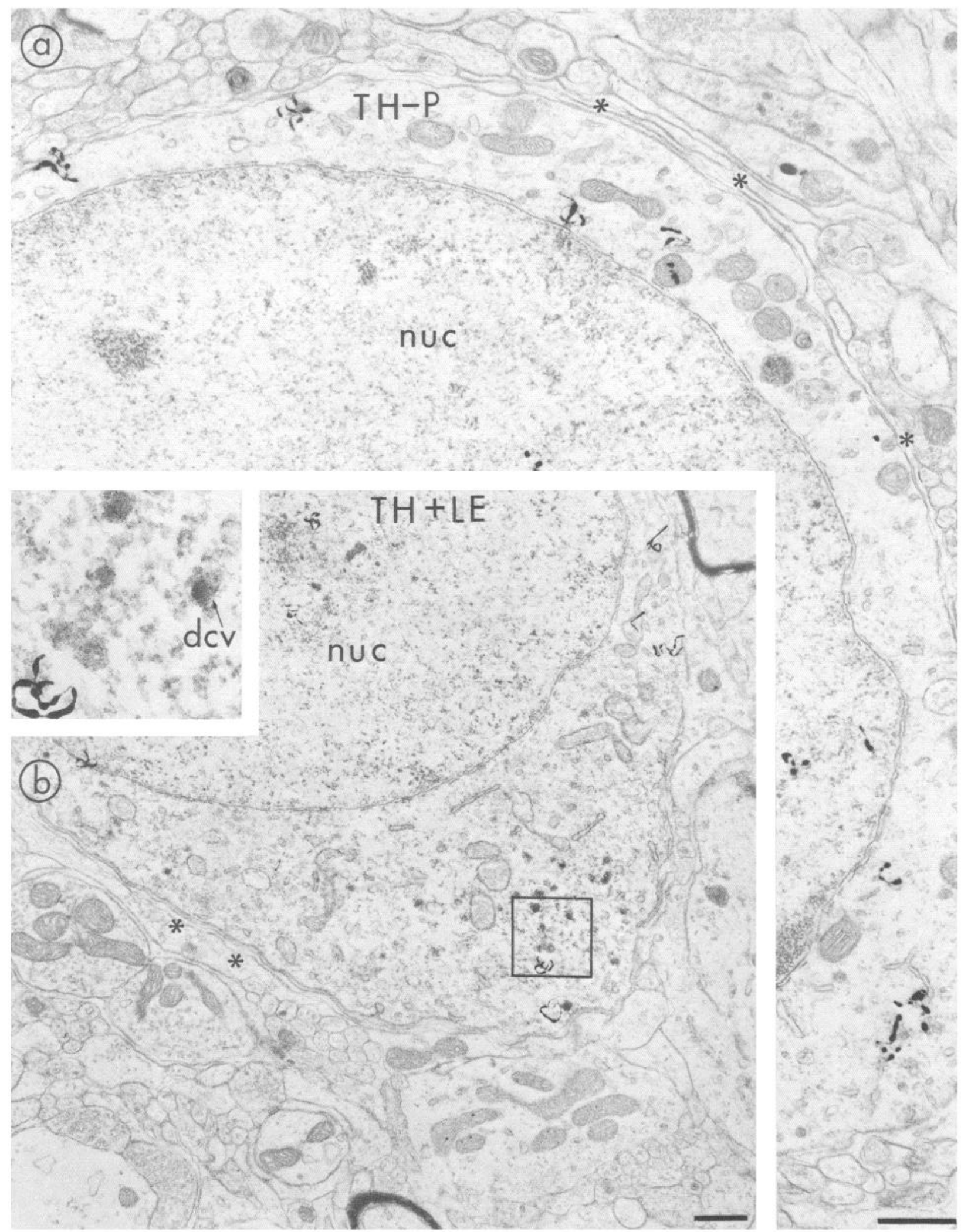

Figure 3. Ultrastructural localization of TH-I and LE-LI in the m-NTS (level 2b). a, Electron micrograph showing silver grains indicative of TH labeling in the cytoplasm of a neuronal perikaryon $(T H-P)$. $b$, Electron micrograph showing the dual localization of LE-LI in large dense-core vesicles in the cytoplasm of a neuron that also contains silver grains indicative of TH-immunoautoradiographic labeling $(T H+L E)$. A few silver grains in both $a$ and $b$ are also seen over the nuclei $(n u c)$ of the respectively labeled perikarya. Inset in the upper left of $b$ shows the boxed region at a higher magnification. Note that the peroxidase product is within the central lumen of the large dense-core vesicles (dcv). Asterisks show the glial lamellae surrounding the labeled perikarya in $a$ and $b$. Scale bar, $0.5 \mu \mathrm{m}$. 

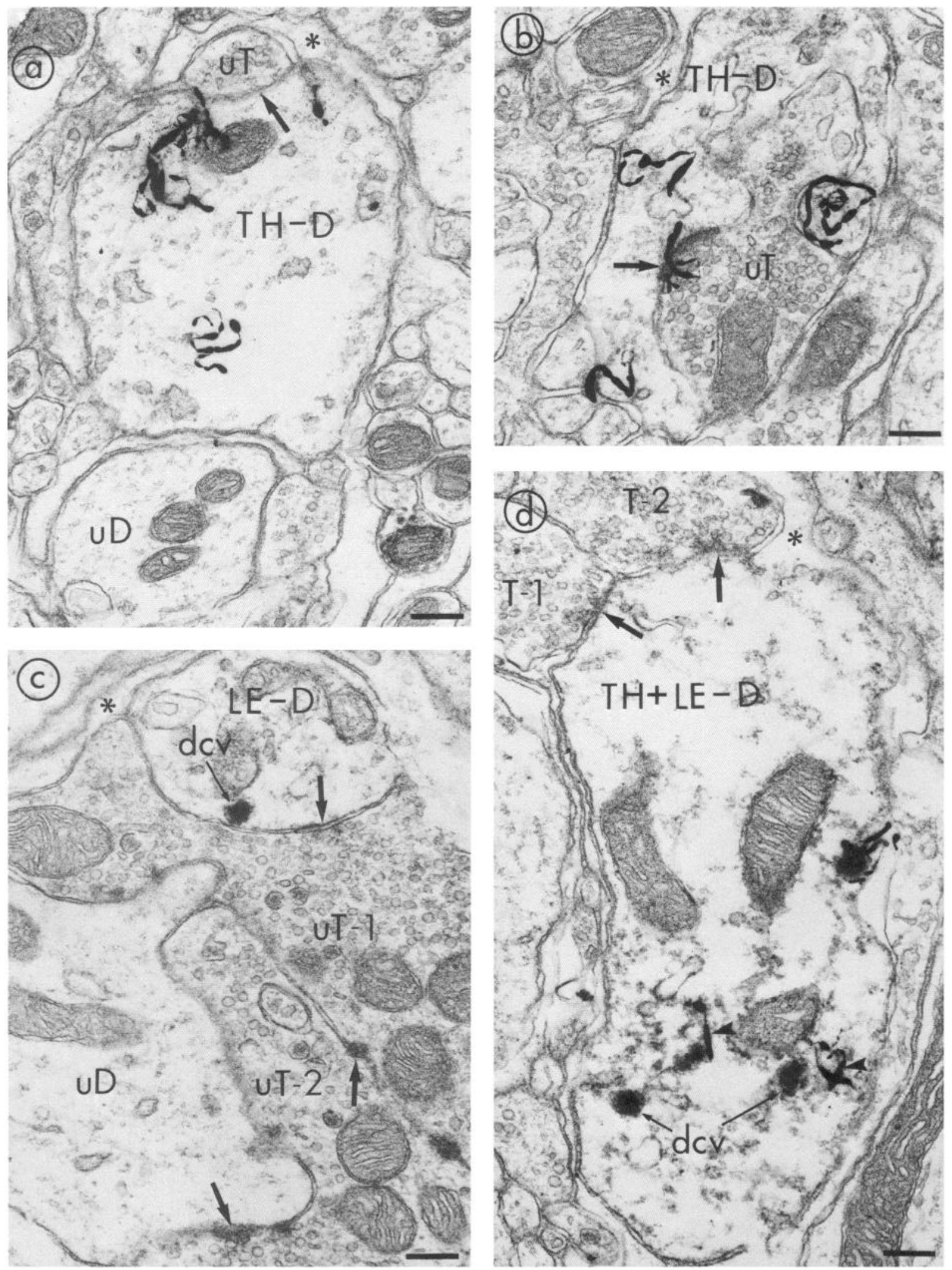

Figure 4. Ultrastructural localization of TH-I and LE-LI in dendrites of the m-NTS (level 2b). $a$, Silver grains indicative of TH labeling are seen within a dendrite $(T H-D)$ and not within the surrounding neuropil or adjacent unlabeled dendrite $(u D)$. The labeled dendrite is in direct apposition (arrow) to a small unlabeled terminal $(u T) . b$, Immunoautoradiographic labeling for TH in a more distal dendritic process $(T H-D)$. The dendrite is postsynaptic to an unlabeled terminal $(u T)$ that forms an asymmetric junction (arrow) with the dendrite. $c$, Peroxidase reaction for LE-LI is seen within a single dense-core vesicle $(d c v)$ in a dendrite labeled LE-D. The dendrite receives a symmetric contact (arrow) from an unlabeled terminal $(u T-1)$. Dense membrane specializations (arrows) also are seen between uT-1 and a second terminal, uT-2, and between uT-2 and an unlabeled 
m-NTS that correspond to the A2-group of catecholaminergic neurons contained greater densities of silver grains (Fig. 1d). This differential labeling was most readily appreciated by the fact that TH-I in the ventral group of neurons was detectable after much shorter autoradiographic exposures.

\section{Electron microscopy}

Electron microscopy of the boxed region of Figure $2 b$ revealed immunoautoradiographic labeling for TH and immunoperoxidase reaction for LE-LI within separate, as well as the same, perikarya, dendrites, and axon terminals. The results confirmed light microscopic findings showing that the majority of the large heavily $\mathrm{TH}$-labeled perikarya were located in ventral portions of the m-NTS at the level of the area postrema. In the dorsal m-NTS at this level, the TH-labeled cells were small and showed a thin rim of cytoplasm with few large dense vesicles or other cytoplasmic organelles (Fig. $3 a$ ). The autoradiographic labeling was seen primarily over the cytoplasm but also was detected over nuclei of most immunolabeled cells. The TH-labeled perikarya were characterized by their relatively sparse synaptic input and extensive glial investments (Fig. 3a). Even in the most superficial sections, the vase majority of these cells did not contain peroxidase labeling for LE-LI. However, a few perikarya having similar morphological features including rounded nuclei, glial investments, and few cytoplasmic organelles were dually labeled. These were detectable only following colchicine treatment (Fig. $3 b$ ). In contrast to the singly labeled cells, the dually labeled perikarya contained more numerous, large $(80-150 \mathrm{~nm})$ dense-core vesicles. The reaction product for LE-LI was principally associated with these vesicles (Fig. $3 b$, inset). Several other perikarya containing LE-LI in dense vesicles lacked autoradiographic labeling for $\mathrm{TH}$, even though nearby processes were labeled for the enzyme.

Dendrites in the m-NTS at the level of the area postrema from colchicine-treated rats also contained either immunoautoradiographic labeling for $\mathrm{TH}$ (Fig. 4, $a, b$ ), peroxidase labeling for LE-LI (Fig. $4 c$ ) or both labels (Fig. $4 d$ ). Autoradiographic silver grains indicative of TH-I were detected in proximal (Fig. 4a) as well as in distal dendrites and spines (Fig. $4 b$ ). These dendrites were extensively invested in glial processes and received few synapses. The presynaptic terminals usually lacked detectable immunoreactivity. In a quantitative evaluation of the types of junctions formed by afferents to TH-labeled dendrites, $90 \%$ from a total of 59 terminals formed symmetric junctions. These included $62 \%$ that were unlabeled, $12 \%$ that were labeled for TH, and $15 \%$ that contained LE-LI. Most of these junctions were on more proximal, large dendrites. Asymmetric junctions principally on distal dendrites and spines accounted for only $10 \%$ of the afferent input. This value may underrepresent the asymmetric junctions on spines due to the greater difficulty of detecting immunoautoradiographic labeling in the smaller distal processes.

In dendrites containing $L E-L I$ (Fig. $4 c$ ) or both $L E-L I$ and $T I I$ (Fig. $4 d$ ), the reaction product was almost exclusively localized to dense-core vesicles. These appeared considerably larger than the unlabeled dense core vesicles seen in nearby axon terminals. Many of these vesicles were located near the dendritic plasmalemma (Fig. 4c). In dually labeled dendrites, the immunoreactive dense-core vesicles sometimes were superimposed with autoradiographic silver grains indicative of $\mathrm{TH}-\mathrm{I}$ (Fig. $4 d$ ). Dendrites containing LE-LI or LE-LI plus TH-I also received relatively few synaptic contacts and were enveloped in glial processes (Fig. 4, $c, d$ ). The detected junctions on these dendrites were principally symmetric and from unlabeled terminals. In favorable planes of section, unlabeled terminals forming symmetric junctions on dendrites containing LE-LI also showed axoaxonic junctions with other unlabeled terminals (Fig. $4 c$ ). These junctions are partially obscured in Figure $4 c$ by the obliqueness of the section.

The TH-labeled terminals were usually $0.5-1.0 \mu \mathrm{m}$ in diameter, although larger $(1.5-2.0 \mu \mathrm{m}) \mathrm{TH}$-labeled terminals also were detected (Fig. 5, $a, b$ ). Small 40-60 nm, round, and clear vesicles were the most prominent organelle in the TH-labeled terminals. Other organelles included mitochondria and one or more large dense-core vesicles (Fig. 5, $a, b$ ). Immunoautoradiographic labeling for TH was established through serial sections of 57 terminals. Of these, $33 \%$ formed symmetric synapses either with unlabeled (26\%) or TH-labeled (7\%) dendrites (see Fig. 10 for schematic representation of the types of associations formed by TH-labeled neurons). Only $2 \%$ formed asymmetric synapses with unlabeled dendrites. The remaining terminals lacked recognized densities within the plane of section. From the total group of TH-labeled terminals, 19\% also contained LE-LI, principally within large dense-core vesicles (Fig. 5, $c, d$ ).

Unmyelinated as well as a few myelinated axons and axon terminals contained LE-LI. In these axons, LE-LI was primarily associated with large dense-core vesicles (Fig. $6 a$ ). The reaction product was seen both within the central lumen of large, densecore vesicles and rimming the smaller vesicles in axon terminals (Figs. 6, $b, c ; 7-9$ ). The PAP-labeled dense core vesicles usually were located at sites distal to the synaptic junctions and near investing glial processes (Fig. 7, $a, b$ ). The glial processes were identified by their cytological characteristics including bundles of intermediate filaments (Peters et al., 1976). In a quantitative evaluation of the same sections used for the analysis of TH-I, 183 terminals showed LE-LI. Many of these formed symmetric junctions. These included junctions on unlabeled (29\%) (Figs. $6, b, c ; 7, a, b)$, TH-labeled (7\%; Fig. 8, $a, b)$, and TH + LELI-labeled ( $3 \%$; Fig. $8 c$ ) soma and dendrites. The cellular associations are schematically depicted in Figure 10. The somatic contacts were rare and exclusively on unlabeled perikarya (Fig. $6, b, c)$. The receptive perikarya included those with electrondense (Fig. $6 b$ ) and electron-lucent cytoplasm. Terminals containing LE-LI occasionally formed symmetric synapses with both an unlabeled soma and an unlabeled dendrite (Fig. 6c). A few terminals containing LE-LI formed symmetric junctions on dendrites that were additionally postsynaptic to a TH-labeled terminal (Fig. 9). These were detected only by careful analysis of serial sections, since the 2 types of terminals often did not both form junctions within the same plane of section.

In contrast to the prevalence of symmetric junctions, only $4 \%$ of the terminals containing LE-LI formed synapses that were

dendrite $(u D)$. $d$, TH-containing dendrite is dually labeled for LE-LI (TH $+L E-D)$. The peroxidase reaction is located within large dense-core vesicles $(d c v)$ and also is diffusely distributed throughout the cytoplasm. Many of the silver grains indicative of immunoautoradiographic labeling for TH also are near the large dense-vesicles (arrowheads). The dendrite receives symmetric junctions (arrows) from 2 unlabeled terminals ( $T 1$ and T2). Asterisks indicate glial processes surrounding the labeled dendrites in $a-d$. Autoradiographic exposure, 4 months. Scale bar, $0.2 \mu \mathrm{m}$ throughout. 

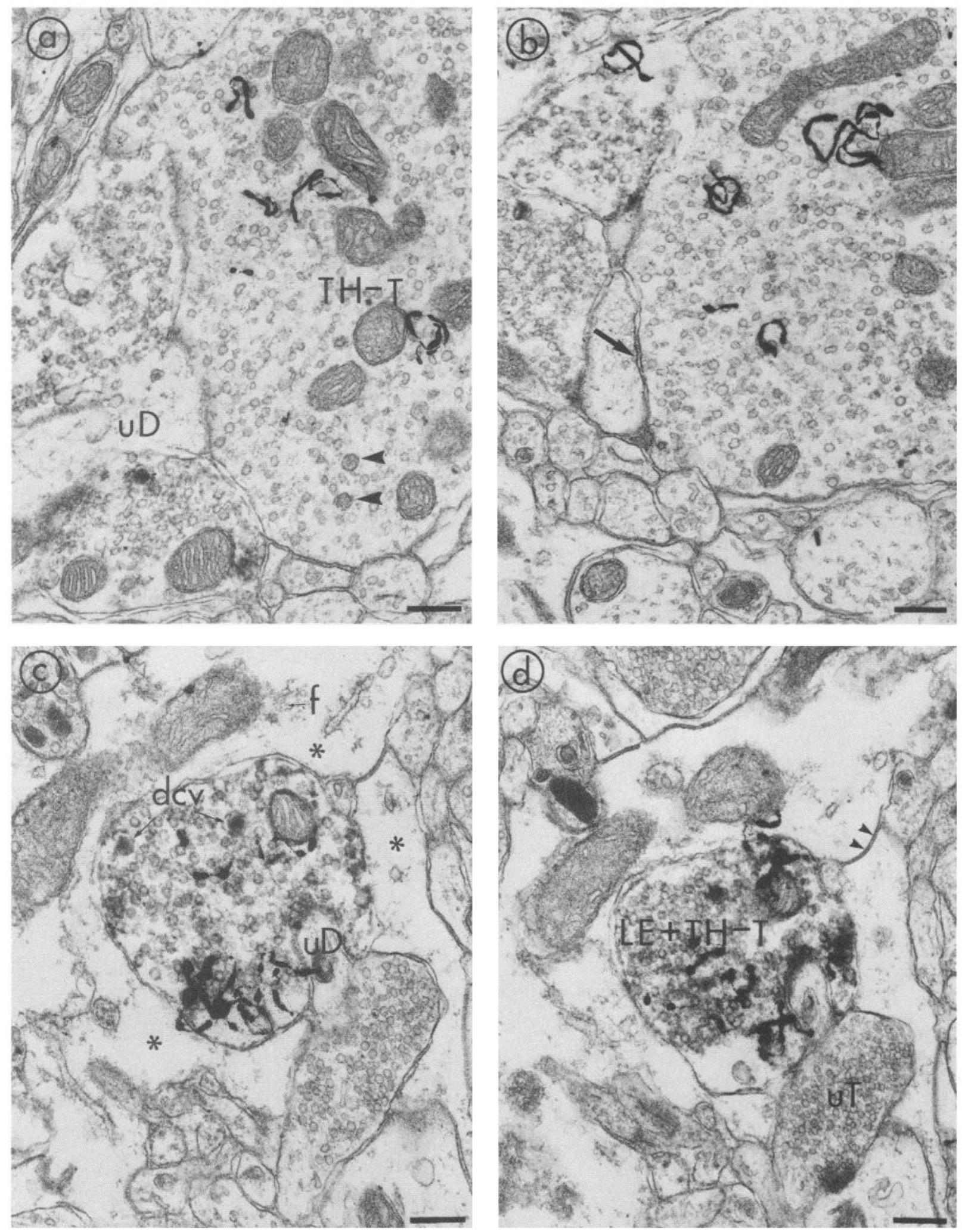

Figure 5. Ultrastructural localization of TH and LE-LI in terminals in the m-NTS (level 2b). $a$ and $b$, Semiadjacent sections showing silver grains that indicate autoradiographic labeling for TH $(T H-T)$. The TH-labeled terminal is in direct apposition (arrow) with an unlabeled dendrite $(u D)$ but lacks clearly defined synaptic densities. Arrowheads show unlabeled dense-core vesicles. $c$ and $d$, Adjacent sections through a terminal labeled for both TH (silver grains) and for LE-LI [peroxidase product rimming small clear vesicles and within large dense-core vesicles $(d c v)$ ]. Compare the density of these dcv with unlabeled dcv in the TH-labeled terminals of $a$ and $b$. The dually labeled terminal forms a synaptic junction with what appears to be an unlabeled small dendritic spine $(u D)$ and is enveloped with glial processes containing bundles of intermediate filaments $(f)$. A tight junction (arrowheads) also joins the 2 glial processes. Another unlabeled terminal $(u T)$ is enveloped within the glial processes. Autoradiographic exposure, 4 months. Scale bar, $0.2 \mu \mathrm{m}$. 

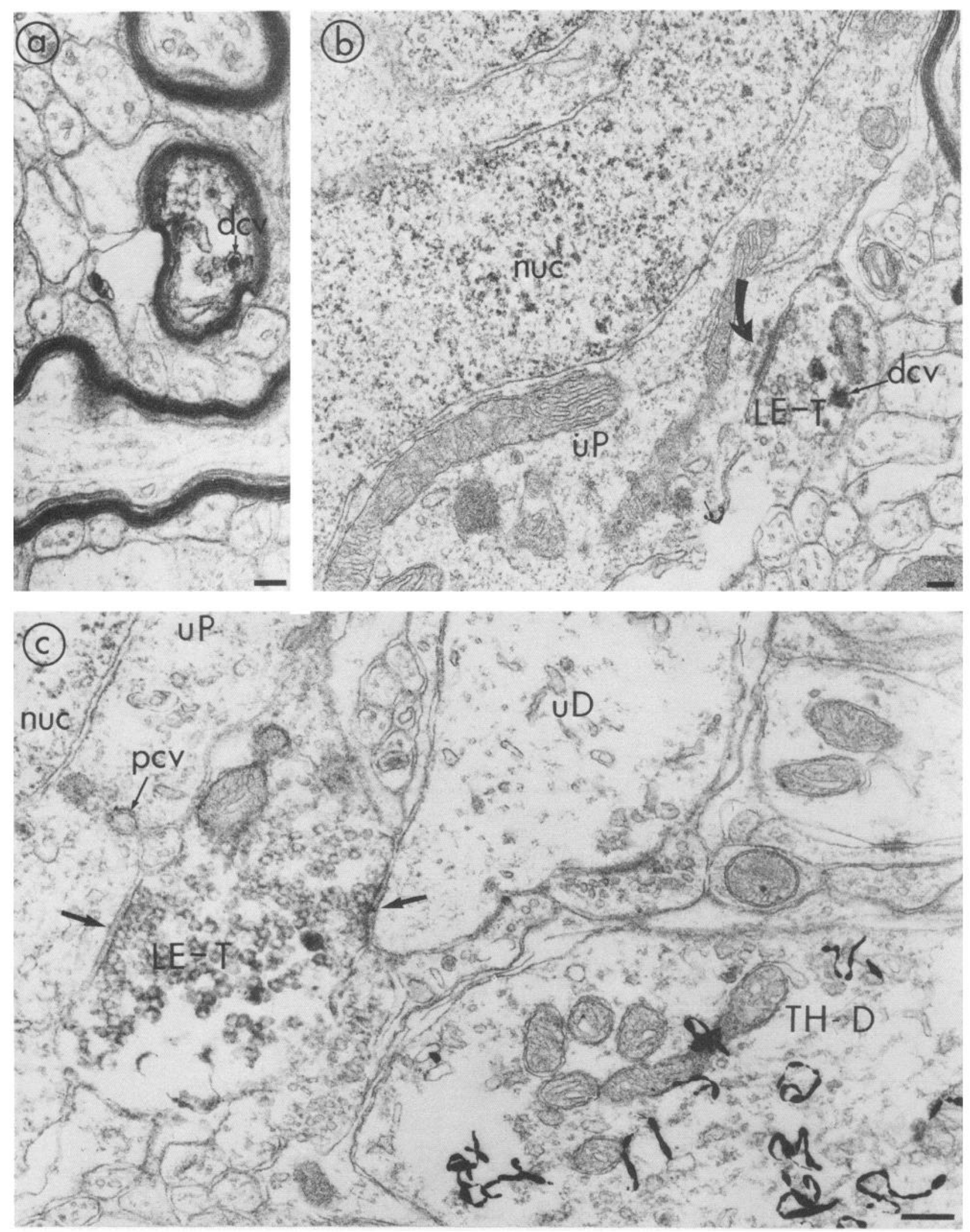

Figure 6. Ultrastructural localization of LE-LI in axons and in terminals forming somatic junctions in the m-NTS (level 2b). $a$, LE-LI is localized to dense-core vesicles $(d c v)$ within a lightly myelinated axon located near the solitary tract. $b$, A somatic junction (curved arrow) is shown between a terminal immunolabeled for LE-LI $(L E-T)$ and an unlabeled perikaryon $(u P)$. The perikaryon has dense cytoplasm but no PAP reaction product and an invaginated nucleus $(n u c)$. In the labeled terminal, the PAP product is localized to the large dense-core vesicles $(d c v)$ but also rims the smaller vesicles. The asymmetric junction seen in this micrograph is not typical of somatic contacts formed by terminals containing LE-LI. $c$, LE$\mathrm{LI}$ is localized to dense-core vesicles and rims smaller vesicles in a terminal forming symmetric junctions (arrows) with both an unlabeled dendrite $(u D)$ and an unlabeled perikaryon $(u P)$. Note that, in addition to the symmetric junction on the soma, a pinocytotic vesicle $(p c v)$ is seen between the nucleus $(n u c)$ and the labeled terminal. A TH-labeled dendrite $(T H-D)$ is also seen in the lower right-hand portion of the figure. Autoradiographic exposure, 4 months. Scale bar, $0.2 \mu \mathrm{m}$. 

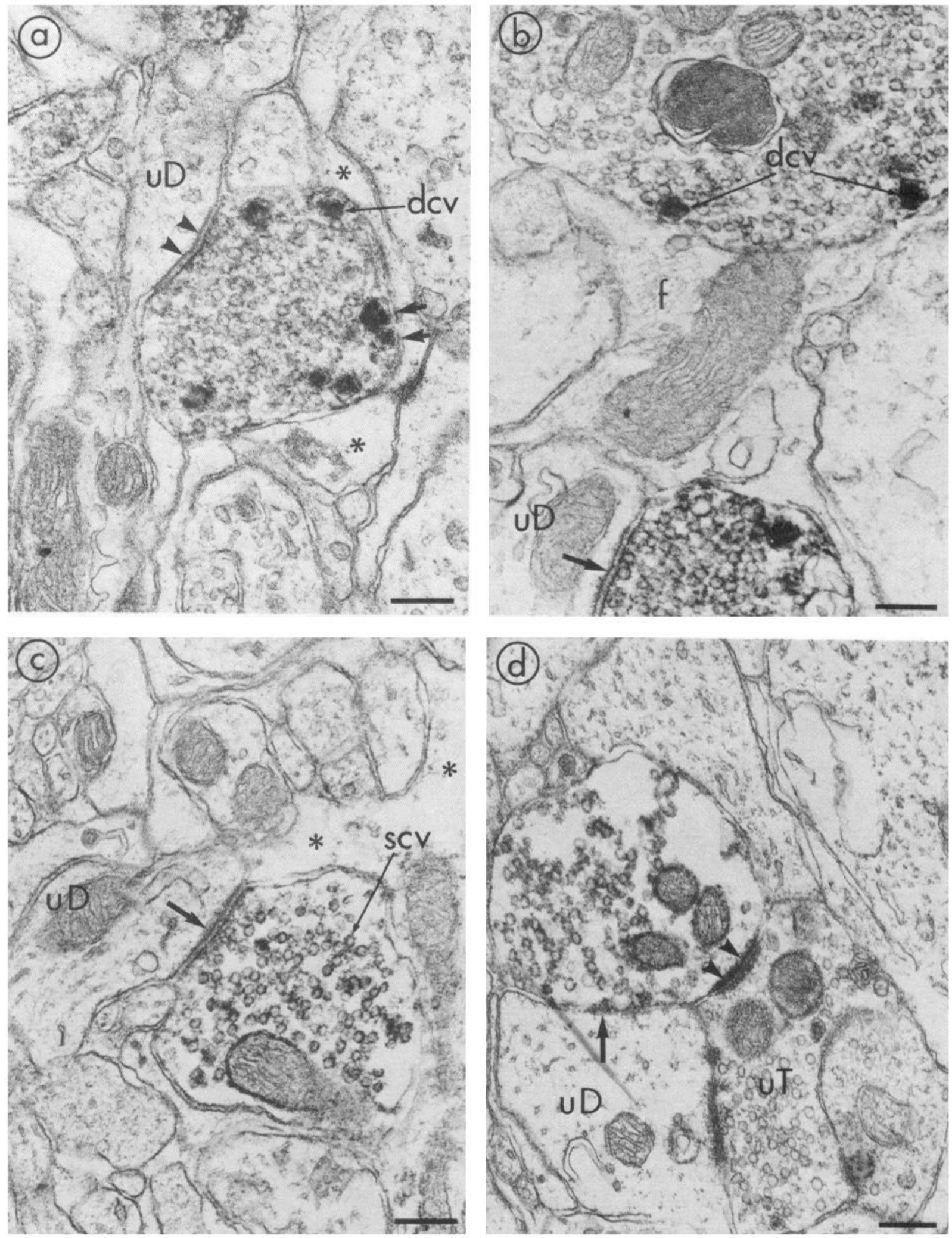

Figure 7. Ultrastructural localization of LE-LI in axon terminals formung axodendritic, glial, and axonic associations. a, Electron micrograph showing the localization of peroxidase reaction for LE-LI principally within large dense-core vesicles $(d c v$ 's). These vesicles are in direct apposition (arrows) to an investing glial process (asterisks). Small clear vesicles are in close proximity to the symmetric junction with the unlabeled dendrite $(u D) . b$, Two terminals containing dense-core vesicle $(d c v)$ with PAP reaction for LE-LI are in direct proximity to a glial process that contains bundles of intermediate filaments $(f)$. The upper terminal formed no recognizable junctions with neuronal processes within the plane of section. However, the lower axon terminal formed a symmetric junction (arrow) with unlabeled dendrite $(u D)$ ). $c$, Terminal showing LE-LI rimming small clear vesicles $(s c v)$ but lacking the larger dense-core vesicles forms an asymmetric junction (arrow) with unlabeled dendrite $(u D)$. Asterisks indicate glial processes surrounding the labeled terminal and dendrite. $d$, Terminal showing LE-LI rimming small clear vesicles is in direct apposition 
clearly asymmetric (Figs. $6 b ; 7 c$ ). These were principally on unlabeled distal dendrites and spines. Axoaxonic junctions with well-defined synaptic specializations also constituted only $1 \%$ of the contacts formed by terminals containing LE-LI (Fig. $7 d$ ). The remaining terminals were without recognized synaptic specializations. From the total number of terminals containing LELI, both with and without recognized targets, $6 \%$ were dually labeled for TH (Fig. 5, c, d).

\section{Discussion}

The findings of this study provide new information of relevance to understanding the cellular relationships between opioid and catecholaminergic as well as noncatecholaminergic neurons and possible astrocytes within the m-NTS. Specifically, we have shown the differential localization and coexistence of LE-LI and $\mathrm{TH}$ and have characterized further the subcellular localization of LE-LI. A quantitative evaluation of sections collected near the surface and, thus equally exposed to all antibodies and immunoreagents, revealed that the terminals containing LE-LI formed symmetric junctions principally, though not exclusively, on noncatecholaminergic neurons, some of which also received innervation from TH-labeled axon terminals.

\section{Light microscopic distributions}

The observation that the intensity of the light microscopic labeling for both TH and LE-LI appeared qualitatively distinct in neighboring perikarya within the same section or within different sections identically processed from different levels of the same brain may reflect several variables. These include the heterogeneity of the catecholamine and opioid transmitters and the quantity or subcellular localization of antigens.

As the enzyme catalyzing the first step in catecholamine synthesis, TH is present within adrenergic, noradrenergic, and dopamincrgic neurons (Joh and Ross, 1983). Thus, variations in labeling intensity may reflect different quantities or solubilities of TH in neurons using these respective transmitters (Pickel, 1986). The caudal two-thirds of the m-NTS contains principally noradrenergic neurons of the A2-cell group (Dahlstrom and Fuxe, 1965). These have a similar distribution to the more heavily TH-labeled perikarya of the ventral group at the level of the area postrema. The lightly TH-labeled perikarya in the dorsal m-NTS at the same level probably correspond to recently identified adrenergic neurons, which also have comparatively low detectable levels of the adrenalin-synthesizing enzyme phenylethanolamine $N$-methyltransferase (PNMT) (Kalia et al., 1985b; Ruggiero et al., 1985). These PNMT-containing neurons have ultrastructural features (Pickel et al., 1986b) comparable to those of the TH-labeled perikarya in the dorsal m-NTS.

Variations in intensity of LE-LI may be attributed to the type of opiate recognized by the monoclonal antibody. The LE antibody was shown in immunodot-blots and adsorption controls to recognize principally LE (Milner et al., 1989). However, crossreaction of the antibody was also noted to a lesser extent with Met $^{5}$-enkephalin; and all dynorphin A fragments (Milner et al., 1989). Thus, the different intensities of labeling may reflect differential detection of $\mathrm{Leu}^{5}$-enkephalin versus other opioid peptides (Larsson et al., 1979). The cross-reactivities suggest that the more darkly labeled perikarya contain LE-LI rather than immunoreactivity for $\mathrm{Met}^{5}$-enkephalin. However, perikarya and processes containing both $\mathrm{Leu}^{5}$ and $\mathrm{Met}^{5}$-enkephalin-like immunoreactivities are seen throughout the rostrocaudal extent of the NTS (Elde et al., 1976; Sar et al., 1978; Maley and Elde, 1982). Thus, both types of enkephalins may have been identified with LE antibody.

Limited accessibility of antibodies to antigens localized principally to vesicles (see next section) also may account for lower levels of detectability of LE-LI in neurons of the caudal m-NTS. This latter difference may be accentuated in acrolein-fixed Vibratome sections, which have more limited penetration than frozen sections of paraformaldehyde-fixed tissues used for most light microscopic immunocytochemical studies (Pickel, 1981). A limited ability to identify dually labeled cells by light microscopy also is likely to be attributable to low levels of antigen and/or localization in vesicles.

\section{Coexistence}

The observed coexistence of LE-LI and TH-I in the same cells is not likely the result of nonspecific cross-reactivity. The absence of nonspecific cross-reactions between $\mathrm{TH}$ antibodies and LE was established by adsorption controls, whereas omission of the second primary antiserum in dually processed sections established absence of nonspecific cross-reactions during subsequent labeling. Moreover, only a few of the cells were labeled for both TH and LE, while numerous cells were singly labeled for each antigen within the same section. Nonspecific interactions would be expected in all immunolabeled cells in sections collected sufficiently close to the surface to allow equal penetration of immunoreagents.

The demonstration of coexistence of LE-LI and TH-I in neurons of the m-NTS supports earlier reports showing the presence of neuropeptides in brain-stem catecholaminergic neurons. Specifically, catecholaminergic neurons in the NTS are known to contain neuropeptide Y (NPY) (Everitt et al., 1984; Harfstrand et al., 1987) and neurotensin (NT; Hökfelt et al., 1984). The distribution of cells dually labeled for catecholamines and for each of these peptides (Hökfelt et al., 1984; Harfstrand et al., 1987) is similar to that of the TH- and LE-containing perikarya in the more caudal m-NTS. It is not known whether these peptides are in the same cells. However, coexistence of enkephalin and somatostatin occurs both in neurons of the m-NTS and in pathways to the NTS from the rostral ventrolateral medulla (Millhorn et al., 1987a,b). Thus, more than one peptide may be present within individual catecholaminergic neurons.

The present demonstration of colocalization of opioid peptides and catecholamines in single neurons within the m-NTS is consistent with earlier light microscopic immunocytochemical studics showing close parallelism between the distribution of opiate-containing tracts and rostrally projecting noradrenergic pathways (Khachaturian and Watson, 1982). However, in this report, the catecholamines and opiates were believed to be contained within separate axons. Our results indicate that a relatively high percentage of the catecholaminergic terminals and a few perikarya in the m-NTS also contain LE-LI. This observation is not surprising in view of the fact that enkephalins 

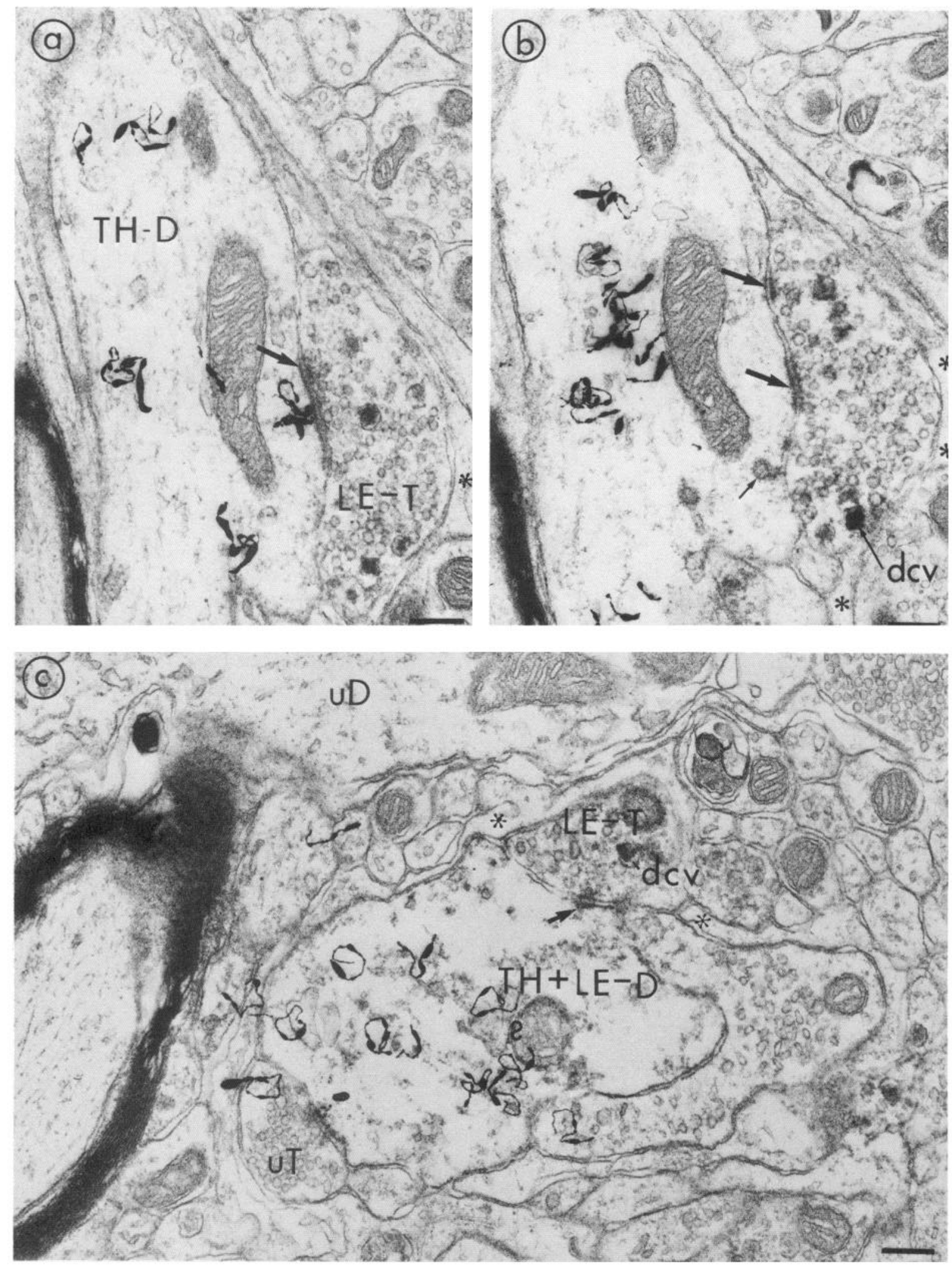

Figure 8. Ultrastructural demonstration of synaptic input from terminals containing LE-LI onto dendrites containing either TH-I or TH-I and LE-LI. $a$ and $b$, Serial sections through the same dendrite and terminal showing immunoautoradiographic labeling for TH (TH-D) and PAP for LE-LI ( $L E-T)$, respectively. Junctions are in patches that appear continuous in a (single arrow) but discontinuous in $b$ (2 arrows). Pinocytotic vesicle (small arrow) is also seen in the TH-D. The labeled dense-core vesicles $(d c v)$ are near investing glial processes (asterisks). $c$, A terminal containing LE-LI $(L E-T)$, principally associated with a large dense-core vesicle $(d c v)$, forms a symmetric synaptic junction (arrow), with a dendrite that contains silver grains indicating immunoautoradiographic labeling for TH and also peroxidase labeling for LE-LI. The dendrite ( $T H+L E$ $D$ ) is labeled diffusely with the PAP reaction product and contains no dense-core vesicles. The dually labeled dendrite also receives synaptic input from another unlabeled terminal $(u T)$. The silver grain over this terminal was not seen in adjacent sections. Autoradiographic exposure, 4 months. Scale bar, $0.2 \mu \mathrm{m}$ throughout. 

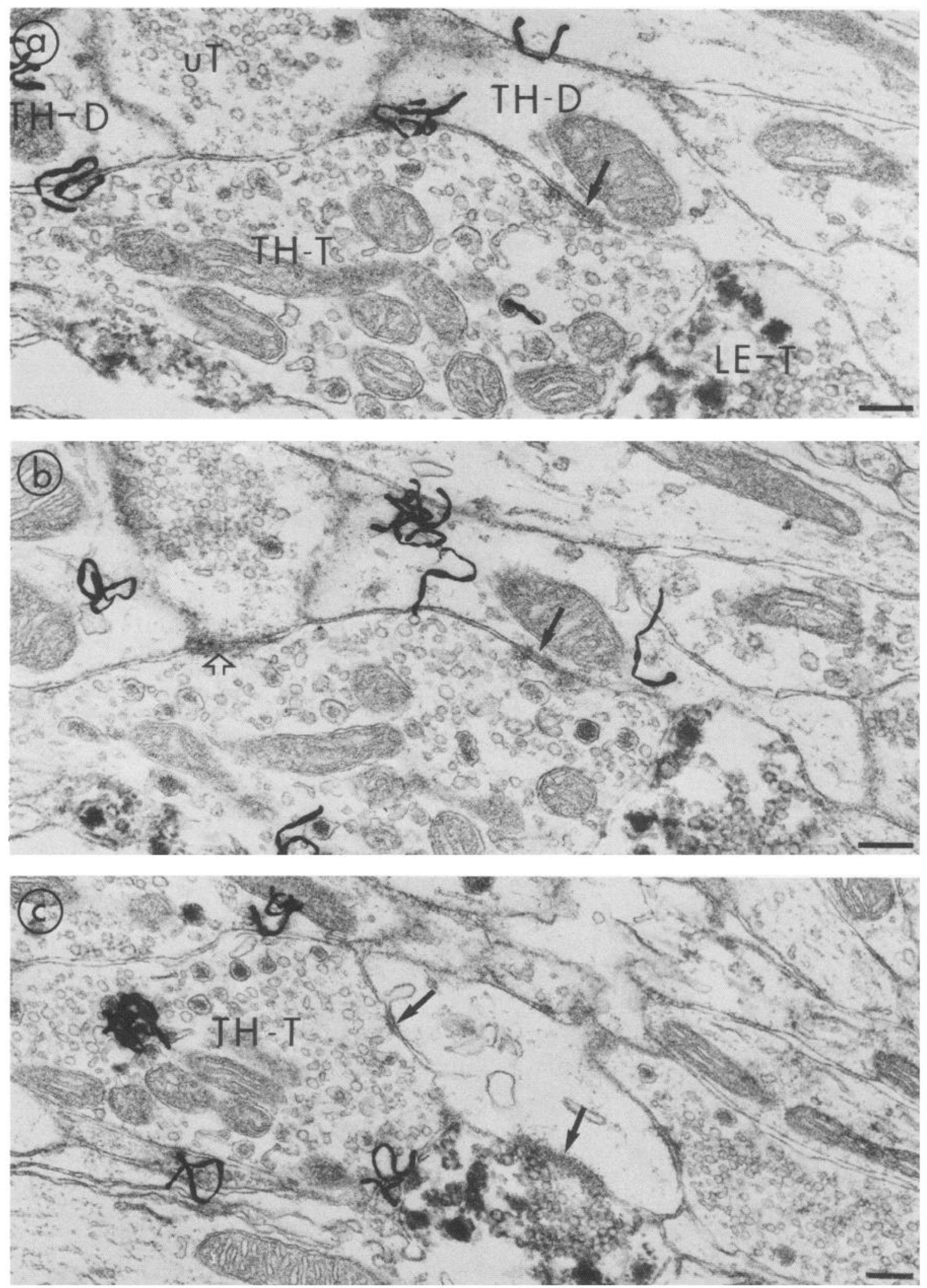

Figure 9. Convergence of separate axon terminals containing TH-I and LE-LI on a common TH-labeled dendrite in the m-NTS. $a-c$, TH-labeled dendrite $(T H-D)$ is seen in three semiadjacent sections with silver grains over the dendrite in 2 of the 3 sections. The TH-labeled terminal $(T H-T)$ shows silver grains in all 3 sections, while the terminal with peroxidase LE-LI (LE-T) also is seen in the 3 sections. Synaptic junctions (arrows) are seen both between the terminals with TH-I and LE-LI and the TH-labeled dendrite. Unlabeled terminal $(u T)$ directly contacts $($ open arrowhead) the TH-I terminal. Autoradiographic exposure, 4 months. Scale bar, $0.2 \mu \mathrm{m}$. 


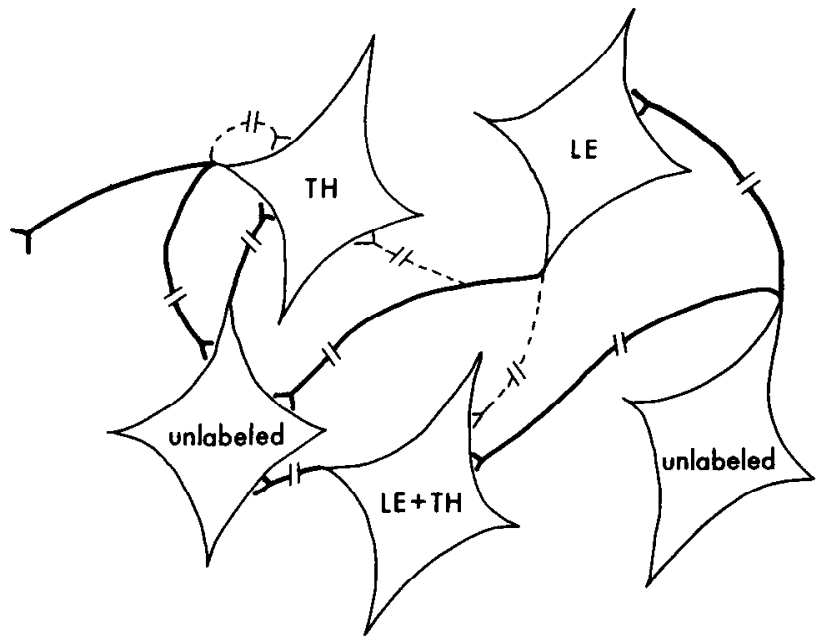

Figure 10. Schematic diagram showing the types of associations formed between neurons containing LE, TH, LE + TH, and unlabeled neurons in the m-NTS (level 2b). Heavy solid lines indicate the more prominent connections, while dashed lines indicate more minor ones. The broken points on the axons indicate the lack of knowledge regarding the origin of the specific afferent. In this schematic, neurons containing TH, LE, and $\mathrm{TH}+\mathrm{LE}$ terminate principally on unlabeled neurons and receive input principally from unlabeled neurons. Less frequently, junctions are seen between LE neurons and neurons containing TH or TH + LE. In addition, TH terminals form synapses with similarly labeled cells. Convergence of TH- and LE-labeled terminals on other unlabeled, as well as TH-labeled, neurons also is depicted.

are costored with catecholamines in chromaffin granules in the adrenal medulla (Viveros et al., 1979). Additionally, noradrenaline coexists with both $\mathrm{Met}^{5}$-enkephalin (De Potter et al., 1987) and with $\mathrm{Leu}^{5}$-enkephalin and dynorphin (Klein et al., 1984) in peripheral sympathetic nerves supplying the vas deferens. Most of the TH-labeled terminals in the present study also are likely to be noradrenergic since norepinephrine is one of the more prominent catecholamines in the intermediate portions of the m-NTS (Kalia et al., 1985a-c). However, we cannot exclude the possibility that some of the terminals may contain adrenalin or possibly dopamine (Kalia et al., 1985a, b).

The terminals immunoreactive for TH, LE-LI, or TH + LELI may be derived from collaterals of local neurons within the rostral or caudal NTS. Another source of these terminals may include the rostral ventrolateral medulla that contains all 3 types of neurons (Milner et al., 1989) and projects to the NTS and spinal cord (Millhorn et al., 1987a). Conversely, perikarya in the NTS containing TH, LE-LI, or TH + LE-LI may contribute to the recently demonstrated pathway from cardiovascular portions of the m-NTS to catecholaminergic neurons in the rostral ventrolateral medulla (Hancock, 1988), the latter neurons receive direct synapses from terminals containing $\mathrm{TH}, \mathrm{LE}$, or $\mathrm{TH}$ + LE (Milner et al., 1989). Such reciprocal interactions between opiate and catecholamine-containing neurons in the NTS and ventral medulla may underlie some of the diverse cardiovascular effects of opiates (Hassen, 1983; Appel et al., 1986).

\section{Subcellular localization}

The demonstration that LE-LI was principally found in large, dense-core vesicles within a few perikarya and dendrites and both within smaller dense-core vesicles and rimming small clear vesicles in axon terminals is analogous to our recent observations with NPY (Pickel et al., 1989a). Maley (1985) also observed similar ultrastructural localization of $\mathrm{Met}^{5}$-enkephalin in cat m-NTS. Moveover, substance P (Pickel et al., 1977; Barber et al., 1979) and other peptides (Pickel, 1985) have a similar distribution in axon terminals in other regions. These findings support the concept that large dense-core vesicles may be the synthesis and storage site for a variety of peptides (Pickel, 1985). However, the large dense-core vesicles additionally may store classic transmitters such as serotonin (Pelletier et al., 1981) or catecholamines (Fried et al., 1985). Some of the large, densecore vesicles, particularly in dendrites of the present study contained both silver grains indicative of TH-I and peroxidase labeling for LE-LI. However, we cannot conclude that the products are colocalized within the same granule for 2 reasons. First, silver grains were seen over both small clear and large dense vesicles in axon terminals. Second, the localization of an ${ }^{125} \mathrm{I}$ autoradiographic label to small organelles requires extensive analysis of central points and distributions of silver grains relative to the organelle since the radiation spread of the isotope is approximately $100 \mathrm{~nm}$ under the conditions used in this study (Salpeter et al., 1977).

The rimming of smaller synaptic vesicles may be attributable to recognition of $L E$, proenkephalins, or other opioid peptides by the LE antibody. These opioids may have diffused from the large or small vesicles during tissue preparation or, alternatively, may never exist within the vesicles (Milner et al., 1989). Absence of immunoreactivity from the central lumen of smaller vesicles also could indicate that the opioids within these vesicles are less accessible to immunoreagents or that these vesicles contain other transmitters (Fried et al., 1985).

\section{Interactions with astrocytes}

Two cellular features observed in this study suggest a particularly close association between neurons containing LE-LI and/ or TH-I and astrocytes in the m-NTS. First, the dense-core vesicles that were most heavily labeled for LE-LI were usually located toward undifferentiated portions of the plasmalemma in direct contact with astrocytic processes. The preferential localization of labeled dense-core vesicles away from synaptic junctions also was reported for Mct $^{5}$-cnkcphalin in terminals of the cat m-NTS (Maley, 1985). Second, terminals labeled either for LE-LI, TH, or both products frequently were enveloped in astrocytic processes, and many failed to form recognizable junctions within the observed sections. The possibility for specific glial actions of catecholamines and opioids is supported by the demonstration that astrocytes have binding sites for these as well as several other putative transmitters (Hosli et al., 1982; Rougon et al., 1983; Bunn et al., 1985). Transmitter-mediated functional changes in astrocytes is supported further by observations that adrenergic agonists alter both the membrane potential and input resistance of cultured glial cells (Hosli et al., 1982). Activation of glial cells through their receptive sites appears to involve either chemically gated ion channels or adenylate cyclase (Rougon et al., 1983; Bernwald-Netter et al., 1986; Hamprecht, 1986). Thus, inhibition of the norepinephrine-stimulated elevation in levels of cyclic AMP by morphinc and by $\mathrm{Met}^{5}$-enkephalin in astrocytic cultures is consistent with dual regulatory mechanisms involving endogenous opiates and catecholamines (Rougon et al., 1983). The functional consequences of interactions of these transmitters with astrocytes, if they occur in the m-NTS, are unknown. However, one possibility is the 
stimulation of astrocytic uptake and metabolism of transmitter amino acids (Hosli et al., 1986). In other regions such as the cerebellum, astrocytic transport of GABA is at least partially regulated by factors produced by neurons having receptive sites for GABA (Drejer et al., 1983). We have recently shown direct synapses between GABAergic terminals and catecholaminergic neurons both in the NTS and in the rostral ventrolateral medulla (Milner et al., 1987; Pickel et al., 1989b). Thus, catecholaminergic neurons probably have receptive sites for GABA and, when stimulated, may release factors that are important for GABA uptake and metabolism by neighboring astrocytes. These factors could be either a catecholamine or coexisting peptide such as LE, NT, or NPY. These may be released by exocytosis into the extracellular space to interact with receptive sites on glia (Golding and Bayraktaroglu, 1984; Zhu et al., 1986; Pow and Golding, 1987; Pickel et al., 1989a). This interesting possibility requires further examination using dual-labeling electron microscopy and in vitro analysis of the capacity of opioids to alter the uptake and metabolism of transmitter amino acids.

\section{Synaptic relations with unlabeled neurons}

Terminals containing LE-LI formed principally symmetric synapses with unlabeled soma and proximal dendrites and more rarely formed asymmetric synapses with unlabeled distal dendrites in the m-NTS. The symmetric versus asymmetric junctions are thought to be associated with inhibition and excitation, respectively (Cohen et al., 1982). Thus, in this context our results indicate that terminals containing LE-LI principally decrease but also may enhance the activity of target neurons, as shown by more direct physiological methods (Zieglgansberger and Tulloch, 1979; Pepper and Henderson, 1980). The 2 types of observed synapses formed by terminals containing LE-LI may reflect different opioid peptides recognized by the LE antibody (Milner et al., 1989) and/or thcir intcractions with differentially specific opioid receptors (Hassen et al., 1983) on proximal versus distal dendrites.

Unlabeled targets of terminals containing LE-LI are likely to include catecholaminergic neurons with nondetectable $\mathrm{TH}$ immunoreactivity. Silver grains indicative of $\mathrm{TH}$ labeling often were sparsely distributed along longitudinally sectioned dendrites. Thus, failure to detect silver grains in serial coronal sections of approximately $60 \mathrm{~nm}$ does not necessarily indicate the absence of TH immunoreactivity. However, the abundance of other neurotransmitters in perikarya and dendrites in the m-NTS strongly suggests that the LE-LI-containing terminals have other noncatecholamine targets. These may include dendrites of cholinergic neurons extending into the m-NTS from the dorsal motor nuclei of the vagus (Armstrong et al., 1988), as well as GABAergic and peptidergic neurons abundantly distributed in cardiovascular portions of the m-NTS (Higgens et al., 1984; Hökfelt et al., 1984; Meeley et al., 1985; Harfstrand et al., 1987).

The observed synaptic junctions between terminals containing LE-LI and unlabeled terminals is consistent with earlier studies showing that opiate receptors occur presynaptically in the striatum (Murrin et al., 1980) and in brain-stem regions receiving somatic (substantia gelatinosa of the spinal trigeminal complex and dorsal horn of the spinal cord) and visceral (NTS) sensory afferents (LaMotte et al., 1976; Atweh and Kuhar, 1977; Atweh et al., 1978). If the unlabeled terminals in direct synaptic contact with terminals containing LE-LI are of peripheral origin, then they are likely to contain substance P (Gillis et al., 1980) or L-glutamate (Talman et al., 1984; Henry and Sessle, 1985).
Presynaptic inhibition of substance $P$ release by opiates is thought to be important in nociceptive pathways in the trigeminal complex (Jessell and Iversen, 1977) and also may be functional in baroreflex pathways in the m-NTS (Maley, 1985). We also cannot exclude other central sources for the unlabeled terminals. One likely possibility is neurotensin-containing neurons within the m-NTS (Higgins et al., 1984). Projections of these neurons to the parabrachial nuclei form axoaxonic contacts that are remarkably similar to the axonic junctions with LE-LI-containing terminals (Milner and Pickel, 1986). Obviously, the unlabeled terminals may contain a variety of other transmitters identified in axon terminals in the m-NTS (Maley and Elde, 1982).

\section{Synaptic input to catecholaminergic neurons}

The major input to catecholaminergic neurons in the m-NTS was from terminals that formed symmetric junctions and lacked detectable levels of either TH or LE-LI. The symmetry of the junctions, abundance of GABA, and functional responses to GABA indicating inhibition of catecholaminergic neurons (Hyden and Cupello, 1987) suggest that GABA may be one of the transmitters in the unlabeled afferents to the TH-I dendrites. More definitive evidence that at least some catecholaminergic dendrites receive afferent input from GABAergic neurons also has been shown by dual labeling (Pickel et al., 1989b). The unlabeled afferents probably also include a variety of neuropeptides, serotonin, and other transmitters identified in terminals in the m-NTS (Higgins et al., 1984; Pickel et al., 1984).

A minor input to $\mathrm{TH}$-labeled dendrites was from terminals containing either LE-LI or TH. The symmetry of the junctions formed by both types of terminals supports physiological data indicating that opiates and alpha $a_{2}$-adrenergic agonists such as clonidine principally inhibit catecholaminergic neurons (Bird and Kuhar, 1977; Aghajanian, 1978; Aghajanian and Wang, 1987). The similarity of the proportions of opioid and catecholaminergic neurons terminating on both unlabeled and THlabeled targets suggests that the convergence may be more common than would be expected by the observed incidence of dual inputs from terminals containing the respective transmitters in the present study. The rarity of detection of this type of convergence is most likely attributed to methodological limitations. The 2 types of terminals were positioned at closely placed sites usually located on small, distal dendrites and dendritic spines. Because of their small size, the spines were difficult to unequivocally demonstrate as autoradiographically labeled for TH. When the afferent terminals were also labeled, the immunoreactivity in small spines may have been missed since the silver grains were thought to originate from radiation spread from the more heavily labeled terminals. (See Salpeter et al., 1977, for discussion of radiation spread using ${ }^{125} \mathrm{I}$.) The controls necessary to establish immunoautoradiographic labeling in small profiles have been discussed at length in some of our more recent papers using the same method (see Pickel et al., 1988; Milner et al., 1989).

Convergent input onto catecholaminergic neurons from terminals with LE-LI and TH in the m-NTS supports evidence that independent stimulation of adrenergic or opioid receptors elicits marked depression of the spontaneous activity of certain groups of noradrenergic neurons (Aghajanian, 1978). More recently, single noradrenergic neurons in the locus coeruleus also have been shown to exhibit tolerance and dependence mediated by potassium channels (Aghajanian and Wang, 1987). The present demonstration of convergent LE-LI and TH-terminals also on noncatecholaminergic neurons in the m-NTS suggests that 
other central neurons may express similar phenomena. Whether convergence as observed in the NTS occurs in other regions more directly involved in opiate withdrawal (Aghajanian, 1978; Kuhar, 1982) and other responses associated with opiates and catecholamines remains to be investigated.

\section{References}

Aghajanian, G. K. (1978) Tolerance of locus coeruleus neurones to morphine and suppression of withdrawal response by clonidine. Nature 276: 186-188.

Aghajanian, G. K., and Y.-Y. Wang (1987) Common alpha-1 and opiate effector mechanisms in the locus coeruleus intracellular studies in brain slices. Neuropharmacology 26: 793-799.

Appel, N. M., J. A. Kiritsy-Roy, and G. R. Van Loon (1986) Mu receptors at discrete hypothalamic and brainstem sites mediate opioid peptide-induced increases in central sympathetic outflow. Brain Res. 378: 8-20.

Armstrong, D. M., A. Rotler, L. B. Hersh, and V. M. Pickel (1988) Localization of choline acetyltransferase in perikarya and dendrites within the nuclei of the solitary tracts. J. Neurosci. Res. 20:279-290.

Atweh, S. F., and M. J. Kuhar (1977) Autoradiographic localization of opiate receptors in rat brain. I. Spinal cord and lower medulla. Brain Res. 124: 53-67.

Atweh, S. F., L. C. Murrin, and M. J. Kuhar (1978) Presynaptic localization of opiate receptors in the vagal and accessory optic systems: An autoradiographic study. Neuropharmacology 17:65-71.

Barber, R. P., J. E. Vaughn, J. R. Slemmon, P. M. Salvaterra, E. Roberts, and S. E. Leeman (1979) The origin, distribution and synaptic relationships of substance $\mathrm{P}$ axons in rat spinal cord. J. Comp. Neurol. 184: 331-352.

Beaudet, A. (1982) High resolution radioautography of central 5-hydroxytryptamine (5-HT). J. Histochem. Cytochem. 30: 765-768.

Berwald-Netter, Y., A. Koulakoff, L. Nowark, and P. Ascher (1986) Ionic channels in glial cells. In Astrocytes, Vol. 2, S. Fedoroff and A. Vernadakis, eds., pp. 51-70, Academic, New York.

Bird, S. J., and M. J. Kuhar (1977) Iontophoretic application of opiates to the locus coeruleus. Brain Res. 122: 523-533.

Bunn, S. J., M. R. Hanley, and G. P. Wilkin (1985) Evidence for a kappa-opioid receptor on pituitary astrocytes: An autoradiographic study. Neurosci. Lett. 55: 317-323.

Cohen, R. S., R. K. Carlin, D. J. Grab, and P. Siekevitz (1982) Phosphoproteins in postsynaptic densities. Prog. Brain Res. 56:49-76.

Dahlström, A., and K. Fuxe (1965) Evidence for the existence of monoamine containing neurons in the central nervous system. I. Demonstration of monoamines in the cell bodies of brain stem neurons. Acta Physiol. Scand. Suppl. 232 62: 1-55.

Dashwood, M. R., M. P. Gilbey, and K. M. Spyer (1985) The localization of adrenoceptors and opiate receptors in regions of the cat central nervous system involved in cardiovascular control. Neuroscience 15: 537-551.

De Potter, W. P., E. P. Coen, and R. W. De Potter (1987) Evidence for the coexistence and corelease of Met-enkephalin and noradrenaline from sympathetic nerves of the bovine vas deferens. Neuroscience 20: 855-866.

Donoghue, S., R. B. Felden, D. Jordan, and K. M. Spyer (1984) The central projections of carotid baroreceptors and chemoreceptors in the cat: A neurophysiological study. J. Physiol. (Lond.) 347: 397410.

Drejer, J., E. Meier, and A. Schousboe (1983) Novel neuron-related regulatory mechanisms for astrocytic glutamate and GABA high-affinity uptake. Neurosci. Lett. 37: 301-306.

Elde, R., T. Hökfelt, O. Johansson, and L. Terenius (1976) Immunohistochemical studies using antibodies to Leucine-enkephalin: Initial observations on the nervous system of the rat. Neuroscience 1 : 340-351.

Everitt, B. J., T. Hökfelt, L. Terenius, K. Takemoto, V. Mutt, and M. Goldstein (1984) Differential coexistence of neuropeptide Y (NPY)like immunoreactivity with catecholamines in the central nervous system of the rat. Neuroscience 11: 443-462.

Fried, R. L., L. Terenius, T. Hökfelt, and M. Goldstein (1985) Evidence for differential localization of noradrenaline and neuropeptide
$Y$ in neuronal storage vesicles isolated from rat vas deferens. J. Neurosci. 5: 450-458.

Gillis, R. A., C. J. Helke, B. L. Hamilton, W. P. Norman, and D. M. Jacobowitz (1980) Evidence that substance $P$ is a neurotransmitter of baro- and chemoreceptor afferents in nucleus tractus solitarii. Brain Res. 181: 476-481.

Golding, D. W., and E. Bayraktaroglu (1984) Exocytosis of secretory granules-A probable mechanism for the release of neuromodulators in invertebrate neuropiles. Experientia 40: 1277-1280.

Granata, A. R., D. A. Ruggiero, D. H. Park, T. H. Joh, and D. J. Reis (1983) Lesions of epinephrine neurons in the rostral ventrolateral medulla abolish vasodepressor components of baroreflex and cardiopulmonary reflex. Hypertension (Suppl. V) 5: 80-84.

Hamprecht, B. (1986) Astroglia cells in culture: Receptors and cyclic nucleotides. In Astrocytes, Vol. 2, S. Fedoroff and A. Vernadakis, eds., pp. 77-107, Academic, New York.

Hancock, M. B. (1988) Evidence for direct projections from the solitary tract onto medullary adrenaline cells. J. Comp. Neurol. 276. $460-467$.

Harfstrand, A, K. Fuxe, L. Terenius, and M. Kalia (1987) Neuropeptide $\mathrm{Y}$-immunoreactive perikarya and nerve terminals in the rat medulla oblongata: Relationship to cytoarchitecture and catecholaminergic cell groups. J. Comp. Neurol. 260: 20-35.

Hassen, A. H., G. Feuerstein, and A. I. Faden (1982) Mu receptors and opioid cardiovascular effects in the nucleus tractus solitarius of rats. Peptides 3: 1031-1037.

Hassen, A. H., G. Feuerstein, and A. I. Faden (1983) Differential cardiovascular effects mediated by $\mathrm{Mu}$ and Kappa opiate receptors in hindbrain nuclei. Peptides 4:621-625.

Henry, J. L., and B. J. Sessle (1985) Effects of glutamate, substance P and eledoisin-related peptide on solitary tract neurons involved in respiration and respiratory reflexes. Neuroscience 14: 863-873.

Higgins, G. A., G. E. Hoffman, S. Wray, and J. S. Schwaber (1984) Distribution of neurotensin-immunoreactivity within baroreceptive portions of the nucleus of the tractus solitarius and dorsal vagal nucleus of the rat. J. Comp. Neurol. 226: 136-164.

Hökfelt, T., K. Fuxe, M. Goldstein, and O. Johansson (1974) Immunohistochemical evidence for the existence of adrenaline neurons in the rat brain. Brain Res. 66: 235-251.

Hökfelt, T., B. J. Everitt, E. Theodorsson-Norheim, and M. Goldstein (1984) Occurrence of neurotensin-like immunoreactivity in subpopulation of hypothalamic, mesencephalic and medullary catecholamine neurons. J. Comp. Neurol. 222: 543-559.

Hosli, E., L. Hosli, and A. Schousboe (1986) Amino acid uptake. In Astrocytes, Vol. 2, S. Fedoroff and A. Vernadakis, eds., pp. 133-153, Academic, New York.

Hosli, L., E. Hosli, C. Zchnter, R. Lehmann, and T. W. Lutz (1982) Evidence for the existence of alpha- and beta-adrenoceptors on cultured glia cells: An electrophysiological study. Neuroscience $7: 2867-$ 2872.

Hyden, H., and A. Cupello (1987) Inhibition in the mammalian brain: A new theory of GABA mechanism of action. Acta Physiol. Scand. Suppl. 561 I30: 1-70.

Jessell, T. M., and L. L. Iversen (1977) Opiate analgesics inhibit substance $P$ release from rat trigeminal nucleus. Nature 268: 549-551.

Joh, T. H., and M. E. Ross (1983) Preparation of catecholamine synthesizing enzymes: An immunogen for immunocytochemistry. In $O x-$ ford IBRO Handbook Series, Vol. 3; Immunocytochemistry, A. C. Cuello, ed., pp. 121-138, Wiley, New York.

Kalia, M., and J. M. Sullivan (1982) Brainstem projections of sensory and motor components of the vagus nerve in the rat. J. Comp. Neurol. 211: 248-264.

Kalia, M., K. Fuxe, and M. Goldstein (1985a) Rat medulla oblongata. II. Dopaminergic, noradrenergic (A1 and A2) and adrenergic neurons, nerve fibers and presumptive terminal processes. J. Comp. Neurol. 233: 308-332.

Kalia, M., K. Fuxe, and M. Goldstein (1985b) Rat medulla oblongata. III. Adrenergic ( $\mathrm{Cl}$ and $\mathrm{C} 2$ ) neurons, nerve fibers and presumptive terminal processes. J. Comp. Neurol. 233: 333-348.

Kalia, M., D. J. Woodward, W. K. Smith, and K. Fuxe (1985c) Rat medulla oblongata. IV. Topographical distribution of catecholaminergic neurons with quantitative three-dimensional computer reconstruction. J. Comp. Neurol. 233: 350-364.

Khachaturian, H., and S. J. Watson (1982) Some perspectives on 
monoamine-opioid peptide interaction in rat central nervous system. Brain Res. Bull. 9: 441-462.

Klein, R. L., S. Lemaire, A. Thureson-Klein, and R. Day (1984) Leuenkephalin, dynorphin and bombesin contents of a highly purified large dense cored noradrenergic vesicle fraction from bovine splenic nerve. In Opioid Peptides in the Periphery, F. Frazoli, A. Esidori, and M. Mazzetti, eds., pp. 205-212, Elsevier, Amsterdam.

Kuhar, M. J. (1982) Receptors for clonidine in brain: Insights into therapeutic actions. J. Clin. Psychiatry 43: 17-19.

LaMotte, C., C. B. Pert, and S. H. Snyder (1976) Opiate receptor binding in primate spinal cord: Distribution and changes after dorsal root section. Brain Res. 112: 407-412.

Larsson, L. I., S. Childers, and S. H. Snyder (1979) Methionine and leucine-enkephalin occur in separate neurons. Nature 22:407-410.

Lipski, J., and E. Solnicka (1976) The effect of activation of central adrenergic receptors by clonidine on the excitability of the solitary tract neurons in cats. Acta Physiol. 27: 309-316.

Maley, B. E. (1985) The ultrastructural localization of enkephalin and substance $\mathbf{P}$ immunoreactivities in the nucleus tractus solitarii of the cat. J. Comp. Neurol. 233: 490-495.

Maley, B. E., and R. Elde (1982) Immunohistochemical localization of putative transmitters within the feline nucleus tractus solitarii. Neuroscience 7: 2469-2490.

Masurovsky, E. R., and R. P. Bunge (1968) Fluorplastic coverslips for long-term nerve-tissue culture. Stain Technol. 43: 161-165.

Meeley, M. P., D. A. Ruggiero, T. Ishitsuka, and D. J. Reis (1985) Intrinsic gamma-aminobutyric acid neurons in the nucleus of the solitary tract and the rostral ventrolateral medulla of the rat: An immunocytochemical and biochemical study. Neurosci. Lett. 58: 8389.

Millhorn, D., K. Seroogy, T. Hökfelt, L. Schmued, L. Terenius, A. Buchan, and J. Brown (1987a) Neurons of the ventrolateral medulla oblongata that contain both somatostatin and enkephalin immunoreactivities project to nucleus solitarii and spinal cord. Brain Res. 424: 99-108.

Millhorn, D., T. Hökfelt, L. Terenius, A. Buchan, and J. C. Brown (1987b) Somatostatin and enkephalin-like immunoreactivities are frequently co-localized in neurons in the caudal brainstem of rat. Exp. Brain. Res. 67: 420-428.

Milner, T. A., and V. M. Pickel (1986) Neurotensin in rat parabrachial region: Ultrastructural localization and extrinsic sources of immunoreactivity. J. Comp. Neurol, 247: 326-343.

Milner, T. A., V. M. Pickel, J. Chan, V. J. Massari, W. H. Oertel, D. H. Park, T. H. Joh, and D. J. Reis (1987) Phenylethanolamine $\mathrm{N}$-methyltransferase-containing neurons in the rostral ventrolateral medulla: II. Synaptic relationships with GABAergic terminals. Brain Res. 411: 46-57.

Milner, T. A., V. M. Pickel, and D. J. Reis (1989) Vetracellular basis for interactions between central opioids and catecholamines: 1 . Rostral ventrolateral medulla. J. Neurosci. 9: 2114-2130.

Murrin, L. C., J. T. Coyle, and M. J. Kuhar (1980) Striatal opiate receptors: Pre- and postsynaptic localization. Life Sci. 27: 1175-1183.

Ordronneau, P., P. B. M. Linstrom, and P. Petrusz (1981) Four unlabeled antibody bridge techniques. A comparison. J. Histochem. Cytochem. 29: 1397-1404.

Paxinos, G., and C. Watson (1986) The Rat Brain in Stereotaxic Coordinates, 2nd ed., Academic, New York.

Pelletier, G., H. W. M. Steinbush, and A. A. J. Verhofstad (1981) Immunoreactive substance $\mathrm{P}$ and serotonin present in the same densecore vesicles. Nature 293: 71-72.

Pepper, C. M., and G. Henderson (1980) Opiates and opioid peptides hyperpolarize locus coeruleus neurons in vitro. Science 209: 394-396.

Peters, A., S. L. Palay, and H. de F. Webster (1976) The Fine Structure of the Nervous System: The Neurons and Supporting Cells, W. B. Saunders, Philadelphia.

Pfeiffer, A., G. Feuerstein, I. J. Kopin, and A. I. Faden (1983) Cardiovascular and respiratory effects of mu-, delta- and kappa-opiate agonists microinjected into the anterior hypothalamic brain area of awake rats. J. Pharmacol. Exp. Ther. 225: 735-741.

Pickel, V. M., (1981) Immunocytochemical methods. In Neuroana- tomical Tract-Tracing Methods, L. Hemier and M. J. Robards, eds., pp. 483-509, Plenum, New York.

Pickel, V. M. (1985) General morphological features of peptidergic neurons. In Handbook of Chemical Neuroanatomy, Vol. 4: GABA and Neuropeptides in the CNS, Part I, A. Bjorklund and T. Hökfelt, eds., pp. 72-92, Elsevier, Amsterdam.

Pickel, V. M. (1986) Ultrastructure of central catecholaminergic neurons. In Neurohistochemistry: Modern Methods and Applications, P. Panula, H. Paivarinta, and S. Soinila, eds., pp. 397-423, Liss, New York.

Pickel, V. M., D. J. Reis, and S. E. Leeman (1977) Ultrastructural localization of substance $\mathbf{P}$ in neurons of rat spinal cord. Brain Res. 122: 534-540.

Pickel, V. M., T. H. Joh, D. J. Reis, S. E. Leeman, and R. J. Miller (1979) Electron microscopic localization of substance $P$ and enkephalin in axon terminals related to dendrites of catecholaminergic neurons. Brain Res. 160: 387-400.

Pickel, V. M., T. H. Joh, J. Chan, and A. Beaudet (1984) Serotonergic terminals: Ultrastructure and synaptic interaction with catecholamine-containing neurons in the medial nuclei of the solitary tracts. J. Comp. Neurol. 225: 291-301.

Pickel, V. M., J. Chan, and T. A. Milner (1986a) Autoradiographic detection of ${ }^{125}$ I-secondary antiserum: A sensitive light and electron microscopic labeling method compatible with peroxidase immunocytochemistry for dual localization of neuronal antigens. J. Histochem. Cytochem. 34: 707-718.

Pickel, V. M., J. Chan, D. H. Park, T. H. Joh, and T. A. Milner (1986b) Ultrastructural localization of phenylethanolamine N-methyltransferase in sensory and motor nuclei of the vagus. J. Neurosci. Res. 15: $439-455$.

Pickel, V. M., J. Chan, and V. J. Massari (1989a) Neuropeptide Y-like immunoreactivity in neurons of the solitary tract nuclei: Vesicular localization and synaptic input from GABAergic terminals. Brain Res. 476: $265-278$.

Pickel, V. M., J. Chan, and T. A. Milner (1989b) Cellular substrates for interactions between neurons containing phenylethanolamine $\mathrm{N}$-methyltransferase and GABA in the nuclei of the solitary tracts. J. Comp. Neurol. (in press).

Pow, D. V., and D. W. Golding (1987) "Neurosecretion" by aminergic synaptic terminals in vitro-A study of secretory granule exocytosis in the corpus cardiacum of the flying locust. Neuroscience 22: 11451149.

Rougon, G., M. Noble, and A. W. Mudge (1983) Neuropeptides modulate the beta-adrenergic response of purified astrocytes in vitro. $\mathrm{Na}$ ture 305: 715-717.

Ruggiero, D. A., C. A. Ross, M. Anwar, D. H. Park, T. H. Joh, and D. J. Rcis (1985) Distribution of neurons containing phenylethanolamine N-methyltransferase in medulla and hypothalamus of rat. J. Comp. Neurol. 239: 127-154.

Salpeter, M. M., H. C. Fertuck, and E. E. Salpeter (1977) Resolution in electron microscope autoradiography. III. Iodine- ${ }^{125}$, the effect of heavy metal staining, and reassessment of critical parameters. J. Cell Biol. 72: 161-173.

Sar, M., W. E. Stumpf, R. J. Miller, K. Chang, and P. Cuatrecasas (1978) Immunohistochemical localization of enkephalin in rat brain and spinal cord. J. Comp. Neurol. 182: 17-38.

Talman, W. T., A. R. Granata, and D. J. Reis (1984) Glutamatergic mechanisms in the nucleus tractus solitarius in blood pressure control. Fed. Proc. 43: 39-44.

Viveros, O. H., E. J. Diliberto, Jr., E. Hazum, and K.-J. Chang (1979) Opiate-like materials in the adrenal medulla: Evidence for storage and secretion with catecholamines. Mol. Pharmacol. 16: 1101-1108.

Zhu, P. C., A. Thureson-Klein, and R. L. Klein (1986) Exocytosis from large dense cored vesicles outside the active synaptic zones of terminals within the trigeminal subnucleus caudalis: A possible mechanism for neuropeptide release. Neuroscience 15: 43-54.

Zieglgansberger, W., and I. F. Tulloch (1979) The effects of methionine- and leucine-enkephalin on spinal neurones of the cat. Brain Res. 167: 53-64. 